\title{
POSITIVE SOLUTIONS OF SEMILINEAR ELLIPTIC EQUATIONS WITH SMALL PERTURBATIONS
}

\author{
RYUJI KAJIKIYA \\ (Communicated by Walter Craig)
}

\begin{abstract}
In this paper, we study the semilinear elliptic equation with a small perturbation. We assume the main term in the equation to have a mountain pass structure but do not suppose any condition for the perturbation term. Then we prove the existence of a positive solution. Moreover, we prove the existence of at least two positive solutions if the perturbation term is nonnegative.
\end{abstract}

\section{INTRODUCTION AND MAIN RESULTS}

We prove the existence of positive solutions for the semilinear elliptic equation

$$
\begin{aligned}
& -\Delta u=f(x, u)+\lambda g(x, u) \text { in } \Omega, \\
& u=0 \quad \text { on } \partial \Omega,
\end{aligned}
$$

where $\Omega$ is a bounded domain in $\mathbb{R}^{N}$ with smooth boundary $\partial \Omega, N \geq 1$ and $f(x, u)$, $g(x, u)$ are continuous on $\bar{\Omega} \times[0, \infty)$ and $\lambda$ is a real parameter whose absolute value is small. We assume a condition on $f(x, u)$ such that (1.1), (1.2) with $\lambda=0$ has a mountain pass structure, and therefore it has a positive solution when $\lambda=0$. The most typical nonlinear term is $f(x, u)=a(x) u^{p}$ or $f(x, u)=a(x) u^{p}+b(x) u^{q}$, where $a, b \in C(\bar{\Omega})$ and $a(x)$ or $b(x)$ may change its sign. The purpose of this paper is to prove the existence of a positive solution for $|\lambda|$ small enough under the mountain pass assumption on $f(x, u)$ only without any conditions on $g(x, u)$. The nonlinear term $f(x, u)=a(x) u^{p}$ was studied by Afrouzi and Brown [1, Alama and Tarantello [2, Brown and Zhang, 4], Li and Wang [6] and the author [5]. However the assumptions in this paper are more general than those of the papers above. We emphasize that our theorem does not need any assumptions on $g(x, u)$. We assume the conditions below.

(f1) There exist positive constants $p, C$ such that $1<p<\infty$ if $N=1,2$ and

$$
1<p<(N+2) /(N-2) \text { if } N \geq 3 \text { and }
$$

$$
|f(x, s)| \leq C\left(s^{p}+1\right) \quad \text { for } s \geq 0, x \in \Omega .
$$

(f2) There exist constants $\alpha>2, \theta \in[0,2), C>0$ such that

$$
\alpha F(x, s)-s f(x, s) \leq C|s|^{\theta}+C \text { for } s \geq 0, x \in \Omega,
$$

Received by the editors August 18, 2011.

2010 Mathematics Subject Classification. Primary 35J20, 35J25, 35J60.

Key words and phrases. Mountain pass lemma, positive solution, perturbation problem, semilinear elliptic equation, variational method.

The author was supported in part by the Grant-in-Aid for Scientific Research (C) (No. 20540197), Japan Society for the Promotion of Science.

(C)2012 American Mathematical Society Reverts to public domain 28 years from publication 
where

$$
F(x, s):=\int_{0}^{s} f(x, t) d t .
$$

(f3) There exist $x_{0} \in \Omega, \delta_{0}>0$ such that

$$
\lim _{s \rightarrow \infty}\left(\min _{\left|x-x_{0}\right| \leq \delta_{0}} F(x, s) / s^{2}\right)=\infty .
$$

$$
\lim \sup _{s \rightarrow 0+}\left(\max _{x \in \bar{\Omega}} f(x, s) / s\right)<\mu_{1},
$$

where $\mu_{1}$ denotes the first eigenvalue of the Dirichlet Laplacian in $\Omega$.

$$
\liminf _{s \rightarrow 0+}\left(\min _{x \in \bar{\Omega}} f(x, s) / s\right)>-\infty .
$$

Assumptions (f1)-(f4) guarantee that $f(x, u)$ has a mountain pass structure, and (f5) ensures that a mountain pass solution is strictly positive. For any $g(x, u)$ and $|\lambda|$ small enough, we prove the existence of a positive solution. Moreover, if $g(x, 0) \geq 0$, we show the existence of another small positive solution.

Theorem 1.1. Let $f(x, s)$ and $g(x, s)$ be continuous on $\bar{\Omega} \times[0, \infty)$. Suppose that (f1)-(f5) hold. Then the following assertions hold.

(i) There exists a $\lambda_{0}>0$ such that (1.1), (1.2) have a positive solution $u_{\lambda}$ when $|\lambda| \leq \lambda_{0}$. Furthermore, for any sequence $\lambda_{j}$ converging to zero, along a subsequence $u_{\lambda_{j}}$, converges to $u_{0}$ in $W^{2, q}(\Omega)$ for all $q \in[1, \infty)$, where $u_{0}$ is a mountain pass solution of (1.1), (1.2) with $\lambda=0$ and where $W^{2, q}(\Omega)$ denotes the Sobolev space.

(ii) If $g(x, 0) \geq 0, \not \equiv 0$ in $\Omega$, then (1.1), (1.2) have another nonnegative solution $v_{\lambda}$ for $\lambda>0$ small enough such that $0 \leq v_{\lambda}(x)<u_{\lambda}(x)$ and $v_{\lambda} \rightarrow 0$ in $W^{2, q}(\Omega)$ as $\lambda \rightarrow 0$ for all $q \in[1, \infty)$. Moreover, if

$$
\liminf _{s \rightarrow 0+}\left(\min _{x \in \bar{\Omega}}(g(x, s)-g(x, 0)) / s\right)>-\infty,
$$

then each $v_{\lambda}$ is strictly positive. if

We give sufficient conditions for (f3)-(f5). Assumptions (f4) and (f5) are fulfilled

$$
\lim _{s \rightarrow 0+} f(x, s) / s=0 \quad \text { uniformly on } \Omega .
$$

Assumption (f3) holds if $f(x, s)$ is superlinear at $s=\infty$ in a small neighborhood of $x_{0}$, i.e.,

$$
\lim _{s \rightarrow \infty}\left(\min _{\left|x-x_{0}\right| \leq \delta_{0}} f(x, s) / s\right)=\infty .
$$

There are many examples of $f(x, s)$ satisfying our assumptions. An easy example of the sign-changing nonlinear term is $f(x, s)=a(x) s^{p}+b(x) s^{q}$, where $a, b \in C(\bar{\Omega})$, $1<q<p$ if $N=1,2$ and $1<q<p<(N+2) /(N-2)$ if $N \geq 3$. The function $f(x, s)$ satisfies (f1)-(f5) if either (i) or (ii) below holds:

(i) $a(x)$ may change its sign, but $a\left(x_{0}\right)>0$ at some $x_{0} \in \Omega$ and $b(x) \leq 0$ in $\Omega$.

(ii) $a(x) \geq 0, \not \equiv 0$ in $\Omega$ and $b(x)$ is any function. 
Indeed, it is easy to verify (f1), (1.4) and (1.5). Let us check (f2). In Case (i), we choose $\alpha=p+1$ so that

$$
(p+1) F(x, s)-s f(x, s)=\frac{p-q}{q+1} b(x) s^{q+1} \leq 0 .
$$

In Case (ii), we choose $\alpha=q+1$ so that

$$
(q+1) F(x, s)-s f(x, s)=\frac{q-p}{p+1} a(x) s^{p+1} \leq 0 .
$$

Thus (f2) holds.

\section{Proof of the Theorem}

We shall prove Theorem 1.1 Our approach is based on the mountain pass lemma and the maximum principle. We always assume (f1)-(f5). Assumptions (f4) and (f5) imply $f(x, 0)=0$. Throughout the paper, we put $f(x, s)=0$ for $s<0$, and hence $f(x, s)$ is defined on $\bar{\Omega} \times \mathbb{R}$ and continuous. Moreover, (f4) and (f5) are still valid as $s \rightarrow 0$ instead of $s \rightarrow 0+$ and (f2) holds for all $s \in \mathbb{R}$.

We call $u$ a solution of (1.1), (1.2) if it belongs to $H_{0}^{1}(\Omega) \cap L^{\infty}(\Omega)$ and satisfies (1.1) in the distribution sense. By the bootstrap argument with the elliptic regularity theorem, $u$ belongs to $W^{2, q}(\Omega)$ for all $q \in[1, \infty)$ and satisfies (1.1) a.e. in $\Omega$. Especially, $u$ lies in $C^{1}(\bar{\Omega})$.

Lemma 2.1. Any nontrivial solution $u$ of (1.1), (1.2) with $\lambda=0$ is strictly positive and $\partial u / \partial \nu<0$ on $\partial \Omega$. Here $\partial / \partial \nu$ denotes the outward normal derivative.

Proof. Let $u$ be a nontrivial solution of (1.1), (1.2) with $\lambda=0$. Put

$$
D:=\{x \in \Omega: u(x)<0\} .
$$

Assume that $D \neq \emptyset$. By the extension of $f(x, s)$ on $s \leq 0$,

$$
-\Delta u=f(x, u)=0 \quad \text { in } D, \quad u=0 \quad \text { on } \partial D .
$$

Thus $u \equiv 0$ in $D$, a contradiction. Therefore $D$ must be empty; i.e., $u \geq 0$ in $\Omega$. Put $A:=\|u\|_{\infty}$. By (f5), there exists a $C>0$ such that $f(x, s) \geq-C s$ for $0 \leq s \leq A$ and $x \in \Omega$. This inequality gives us

$$
(C-\Delta) u=C u+f(x, u) \geq 0 \quad \text { in } \Omega .
$$

By the Hopf maximum principle, $u$ is strictly positive and $\partial u / \partial \nu<0$ on $\partial \Omega$.

For (1.1) with $\lambda=0$, we define the Lagrangian functional $I_{0}(u)$ by

$$
I_{0}(u):=\int_{\Omega}\left(\frac{1}{2}|\nabla u|^{2}-F(x, u)\right) d x,
$$

where $F(x, u)$ is defined in (f2). In what follows, $\|\cdot\|_{p}$ denotes the $L^{p}(\Omega)$ norm. $H_{0}^{1}(\Omega)$ stands for the usual Sobolev space equipped with the norm $\|u\|_{H_{0}^{1}(\Omega)}:=$ $\|\nabla u\|_{2}$. Because of (f1), $I_{0}$ is well defined in $H_{0}^{1}(\Omega)$ and becomes a $C^{1}$ functional.

Lemma 2.2. $I_{0}$ satisfies the Palais-Smale condition.

Proof. Let $u_{n}$ be any sequence in $H_{0}^{1}(\Omega)$ such that $I_{0}\left(u_{n}\right)$ is bounded and $\left\|I_{0}^{\prime}\left(u_{n}\right)\right\|$ converges to zero. From an easy calculation, we see that

$$
I_{0}^{\prime}(u) u=\int_{\Omega}\left(|\nabla u|^{2}-u f(x, u)\right) d x,
$$


which shows that

$$
\begin{aligned}
& \alpha I_{0}\left(u_{n}\right)-I_{0}^{\prime}\left(u_{n}\right) u_{n} \\
& \quad=\frac{\alpha-2}{2}\left\|\nabla u_{n}\right\|_{2}^{2}-\int_{\Omega}\left(\alpha F\left(x, u_{n}\right)-u_{n} f\left(x, u_{n}\right)\right) d x .
\end{aligned}
$$

Hereafter we assume $\theta \geq 1$ in (f2) because in case $\theta<1$ we replace $\theta$ by 1 and $C$ by a larger constant. Then the norm $\|\cdot\|_{\theta}$ makes sense. Since $\left|I_{0}\left(u_{n}\right)\right|$ and $\left\|I_{0}^{\prime}\left(u_{n}\right)\right\|$ are bounded, we use (f2) to get a constant $C>0$ such that

$$
\begin{aligned}
\frac{\alpha-2}{2}\left\|\nabla u_{n}\right\|_{2}^{2} & =\alpha I_{0}\left(u_{n}\right)-I_{0}^{\prime}\left(u_{n}\right) u_{n}+\int_{\Omega}\left(\alpha F\left(x, u_{n}\right)-u_{n} f\left(x, u_{n}\right)\right) d x \\
& \leq C+C\left\|\nabla u_{n}\right\|_{2}+C\left\|u_{n}\right\|_{\theta}^{\theta} \\
& \leq C+C\left\|\nabla u_{n}\right\|_{2}+C^{\prime}\left\|\nabla u_{n}\right\|_{2}^{\theta},
\end{aligned}
$$

where we have used the Sobolev embedding. Since $\theta<2,\left\|\nabla u_{n}\right\|_{2}$ is bounded. Then a subsequence of $u_{n}$ weakly converges in $H_{0}^{1}(\Omega)$. This convergence becomes a strong one, which can be proved in the standard method. See [3, 7, 8, 9] for the details. The proof is complete.

Lemma 2.3. $I_{0}$ has a mountain pass geometry; i.e., there exist $u_{1} \in H_{0}^{1}(\Omega)$ and constants $r, \rho>0$ such that $I_{0}\left(u_{1}\right)<0,\left\|\nabla u_{1}\right\|_{2}>r$ and

$$
I_{0}(u) \geq \rho \quad \text { when }\|\nabla u\|_{2}=r .
$$

Proof. Recall that (f4) is still valid as $s \rightarrow 0$ instead of $s \rightarrow 0+$. Then we have $s_{0}>0$ and $\mu \in\left(0, \mu_{1}\right)$ such that

$$
f(x, s) / s<\mu \text { for }|s|<s_{0},
$$

which implies that

$$
F(x, s) \leq(\mu / 2) s^{2} \text { for }|s| \leq s_{0} .
$$

This inequality with (f1) shows that

$$
F(x, s) \leq(\mu / 2) s^{2}+C|s|^{p+1} \quad \text { for } s \in \mathbb{R},
$$

with some $C>0$. Since $\mu_{1}$ is the first eigenvalue of $-\Delta$, it follows that $\|\nabla u\|_{2}^{2} \geq$ $\mu_{1}\|u\|_{2}^{2}$ for $u \in H_{0}^{1}(\Omega)$. Then $I_{0}$ is estimated as

$$
I_{0}(u) \geq \frac{1}{2}\|\nabla u\|_{2}^{2}-\frac{\mu}{2}\|u\|_{2}^{2}-C\|u\|_{p+1}^{p+1} \geq \frac{\mu_{1}-\mu}{2 \mu_{1}}\|\nabla u\|_{2}^{2}-C^{\prime}\|\nabla u\|_{2}^{p+1} .
$$

This shows the existence of $r$ and $\rho$ satisfying (2.2). Let $x_{0}, \delta_{0}$ be as in (f3). Let $\phi$ be a function such that $\phi \in C_{0}^{1}(\Omega), \phi \geq 0, \phi \not \equiv 0$ and the support of $\phi$ is in $B\left(x_{0}, \delta_{0}\right)$. Here $B\left(x_{0}, \delta_{0}\right)$ is a ball centered at $x_{0}$ with radius $\delta_{0}$. By (f3),

$$
\min \left\{F(x, s) / s^{2}: x \in \overline{B\left(x_{0}, \delta_{0}\right)}\right\} \rightarrow \infty \quad \text { as } s \rightarrow \infty .
$$

Put $a:=\|\phi\|_{\infty} / 2$ and

$$
D:=\left\{x \in B\left(x_{0}, \delta_{0}\right): \phi(x) \geq a\right\} .
$$

For $t \geq 0$, we compute

$$
\begin{aligned}
I_{0}(t \phi) & =\left(t^{2} / 2\right)\|\nabla \phi\|_{2}^{2}-\int_{\Omega} F(x, t \phi) d x \\
& \leq\left(t^{2} / 2\right)\|\nabla \phi\|_{2}^{2}-t^{2} \int_{D} \frac{F(x, t \phi)}{t^{2} \phi^{2}} \phi^{2} d x \rightarrow-\infty \quad \text { as } t \rightarrow \infty .
\end{aligned}
$$


We fix $t>0$ so large that $I_{0}(t \phi)<0$ and $t\|\nabla \phi\|_{2}>r$. Then $u_{1}:=t \phi$ satisfies the assertion of the lemma.

For $u_{1}$ in Lemma 2.3, we define

$$
\begin{gathered}
\Gamma:=\left\{\gamma \in C\left([0,1], H_{0}^{1}(\Omega)\right): \gamma(0)=0, \gamma(1)=u_{1}\right\}, \\
c_{0}:=\inf _{\gamma \in \Gamma} \max _{0 \leq t \leq 1} I_{0}(\gamma(t)) .
\end{gathered}
$$

Lemma 2.4. $c_{0}$ is a critical value of $I_{0}$.

Proof. This is a well-known mountain pass lemma. For the proof, we refer the reader to [3, 7, 8, 9 .

We call $u$ a mountain pass solution of $I_{0}$ if $I_{0}^{\prime}(u)=0$ and $I_{0}(u)=c_{0}$. In general, a mountain pass solution is not necessarily unique but we have an a priori estimate for all mountain pass solutions in the next lemma.

Lemma 2.5. There exists a constant $C>0$ such that $\|u\|_{C^{1}(\bar{\Omega})} \leq C$ for any mountain pass solution $u$ of $I_{0}$.

Proof. Let $u$ be any mountain pass solution of $I_{0}$. Since $I_{0}^{\prime}(u)=0$ and $I_{0}(u)=c_{0}$, we use (2.1) with (f2) to get

$$
\frac{\alpha-2}{2}\|\nabla u\|_{2}^{2} \leq \alpha c_{0}+C\|u\|_{\theta}^{\theta}+C \leq \alpha c_{0}+C^{\prime}\|\nabla u\|_{2}^{\theta}+C .
$$

This gives an a priori bound of the $H_{0}^{1}(\Omega)$ norm of $u$; i.e., $\|\nabla u\|_{2} \leq C$ with a $C>0$ independent of $u$. By the bootstrap argument with (f1) and the elliptic regularity theorem, we get the upper bound of the $W^{2, q}(\Omega)$ norm of $u$ for all $q \in[1, \infty)$. Especially, an a priori $C^{1}(\bar{\Omega})$ estimate of $u$ follows.

By Lemma 2.5, we have an $M>0$ such that

$$
\|u\|_{\infty} \leq M \text { for any mountain pass solution } u \text { of } I_{0} .
$$

Now, we define

$$
\widetilde{g}(x, s)= \begin{cases}g(x, 0) & \text { if } s \leq 0, \\ g(x, s) & \text { if } 0 \leq s \leq 2 M, \\ g(x, 2 M) & \text { if } s \geq 2 M .\end{cases}
$$

Then $\widetilde{g}(x, s)$ is continuous and bounded on $\bar{\Omega} \times \mathbb{R}$. We choose a function $h \in C_{0}^{\infty}(\mathbb{R})$ such that $0 \leq h \leq 1$ in $\mathbb{R}, h(s)=1$ for $|s| \leq 2 M$ and $h(s)=0$ for $|s| \geq 4 M$. We define

$$
\begin{gathered}
I_{\lambda}(u):=\int_{\Omega}\left(\frac{1}{2}|\nabla u|^{2}-F(x, u)-\lambda h(u) \widetilde{G}(x, u)\right) d x, \\
\widetilde{G}(x, u):=\int_{0}^{u} \widetilde{g}(x, s) d s .
\end{gathered}
$$

A critical point of $I_{\lambda}$ is a solution of

$$
-\Delta u=f(x, u)+\lambda h(u) \widetilde{g}(x, u)+\lambda h^{\prime}(u) \widetilde{G}(x, u) \text { in } \Omega,
$$

with $u=0$ on $\partial \Omega$. Our plan to prove Theorem 1.1 is as follows. First, we find a mountain pass solution $u_{\lambda}$ of $I_{\lambda}$. Next, we prove that $0<u_{\lambda}(x) \leq 2 M$ for $|\lambda|$ small enough. Then $h^{\prime}\left(u_{\lambda}\right)=0, h\left(u_{\lambda}\right)=1, \widetilde{g}\left(x, u_{\lambda}\right)=g\left(x, u_{\lambda}\right)$ and therefore $u_{\lambda}$ becomes a solution of (1.1), (1.2). 
Using the same argument as in Lemma 2.2 with the fact that $h(s) \widetilde{G}(x, s)$ and its partial derivative on $s$ are bounded, we get the next lemma.

Lemma 2.6. For each $\lambda \in \mathbb{R}, I_{\lambda}$ satisfies the Palais-Smale condition.

Lemma 2.7. There exists a $\lambda_{0}>0$ such that $I_{\lambda}$ has a mountain pass geometry when $|\lambda| \leq \lambda_{0}$.

Proof. Since $h(s) \widetilde{G}(x, s)$ is bounded on $\bar{\Omega} \times \mathbb{R}$, we have

$$
I_{0}(u)-|\lambda| C \leq I_{\lambda}(u) \leq I_{0}(u)+|\lambda| C \quad \text { for } u \in H_{0}^{1}(\Omega),
$$

where $C>0$ is independent of $\lambda$ and $u$. Let $r, \rho$ and $u_{1}$ be as in Lemma 2.3. For $|\lambda|$ small enough, it follows that

$$
\begin{gathered}
I_{\lambda}\left(u_{1}\right) \leq I_{0}\left(u_{1}\right)+|\lambda| C<0, \\
I_{\lambda}(u) \geq \rho-|\lambda| C \geq \rho / 2 \quad \text { when }\|\nabla u\|_{2}=r .
\end{gathered}
$$

The proof is complete.

We define the mountain pass value $c_{\lambda}$ of $I_{\lambda}$ by

$$
c_{\lambda}:=\inf _{\gamma \in \Gamma} \max _{0 \leq t \leq 1} I_{\lambda}(\gamma(t)) .
$$

Then $c_{\lambda} \rightarrow c_{0}$ as $\lambda \rightarrow 0$ by (2.5).

Lemma 2.8. Let $\lambda_{n} \in \mathbb{R}$ be a sequence converging to zero and $u_{n}$ a mountain pass solution of $I_{\lambda_{n}}$. Then a subsequence of $u_{n}$ converges to a limit $u_{0}$ in $W^{2, q}(\Omega)$ for all $q \in[1, \infty)$, where $u_{0}$ is a mountain pass solution of $I_{0}$.

Proof. By definition, $I_{\lambda_{n}}\left(u_{n}\right)=c_{\lambda_{n}}, I_{\lambda_{n}}^{\prime}\left(u_{n}\right)=0$ and hence $u_{n}$ satisfies (2.4) with $\lambda$ replaced by $\lambda_{n}$. Using the same argument as in Lemma 2.5 with the boundedness of $c_{\lambda_{n}}$, we can prove that the $W^{2, q}(\Omega)$ norm of $u_{n}$ is bounded for any $q \in[1, \infty)$. By the compact embedding, a subsequence of $u_{n}$ converges to a limit $u_{0}$ in $C^{1}(\bar{\Omega})$. Then $u_{0}$ satisfies that $I_{0}\left(u_{0}\right)=c_{0}$ and $I_{0}^{\prime}\left(u_{0}\right)=0$, i.e., that $u_{0}$ is a mountain pass solution of $I_{0}$. The right-hand side of (2.4) with $u=u_{n}$ and $\lambda=\lambda_{n}$ converges to that with $u=u_{0}$ and $\lambda=0$ uniformly on $x \in \bar{\Omega}$. The elliptic regularity theorem again ensures that $u_{n}$ converges to $u_{0}$ strongly in $W^{2, q}(\Omega)$ for all $q \in[1, \infty)$.

We shall prove the positivity and a priori estimate of mountain pass solutions for $I_{\lambda}$. To this end, for $\delta>0$, we put

$$
\Omega_{\delta}:=\{x \in \Omega: \operatorname{dist}(x, \partial \Omega)<\delta\},
$$

where $\operatorname{dist}(x, \partial \Omega)$ denotes the distance from $x$ to $\partial \Omega$.

Lemma 2.9. There exist constants $\lambda_{0}, \delta, a, b>0$ such that any mountain pass solution $u$ of $I_{\lambda}$ with $|\lambda| \leq \lambda_{0}$ satisfies (i) and (ii) below.

(i) $0<u(x) \leq 2 M$ in $\Omega$, where $M$ has been defined by (2.3).

(ii) $\partial u / \partial \nu<-a$ in $\Omega_{\delta}$ and $u(x)>b$ in $\Omega \backslash \Omega_{\delta}$. Here $\partial / \partial \nu$ is well defined at each point in $\Omega_{\delta}$ for $\delta>0$ small because $\partial \Omega$ is smooth.

Proof. First, we shall prove $|u(x)| \leq 2 M$ for $|\lambda|>0$ small enough. Suppose that our claim is false. Then there exist sequences $\lambda_{n} \in \mathbb{R}$ and $u_{n}$ such that $\lambda_{n}$ converges to zero, $u_{n}$ is a mountain pass solution of $I_{\lambda_{n}}$ and $\left\|u_{n}\right\|_{\infty}>2 M$. By Lemma 2.8. a subsequence of $u_{n}$ converges to a mountain pass solution $u_{0}$ of $I_{0}$ in $C^{1}(\bar{\Omega})$. Since $\left\|u_{0}\right\|_{\infty} \leq M$ by (2.3), it follows that $\left\|u_{n}\right\|_{\infty}<2 M$ for $n$ large enough. A 
contradiction occurs. Thus we have $\|u\|_{\infty} \leq 2 M$. The positivity of $u$ in (i) follows from (ii).

Next, we shall prove that $\partial u / \partial \nu<-a$ in $\Omega_{\delta}$ with some $a, \delta>0$ independent of $u$. Suppose on the contrary that there exist $\lambda_{n}, x_{n}, u_{n}$ such that $\lambda_{n} \rightarrow 0$, $\operatorname{dist}\left(x_{n}, \partial \Omega\right) \rightarrow 0, u_{n}$ is a mountain pass solution of $I_{\lambda_{n}}$ and

$$
\liminf _{n \rightarrow \infty} \partial u_{n}\left(x_{n}\right) / \partial \nu \geq 0 .
$$

We choose a subsequence of $x_{n}$ which converges to a limit $x_{0} \in \partial \Omega$. By Lemma 2.8, a subsequence of $u_{n}$ converges to a mountain pass solution $u_{0}$ of $I_{0}$ in $C^{1}(\bar{\Omega})$. Then $\partial u_{0} / \partial \nu\left(x_{0}\right) \geq 0$, a contradiction to Lemma 2.1. Thus $\partial u / \partial \nu<-a$ in $\Omega_{\delta}$ with some $a, \delta>0$. Fix such a $\delta>0$. Then by the same method as above, we can prove that $u(x)>b$ in $\Omega \backslash \Omega_{\delta}$ with some $b>0$.

In Lemma 2.3, we replace $r$ by any positive constant smaller than $r$. Then (2.2) is still valid after $\rho$ is replaced by a smaller positive constant. Hence (2.6) still holds if $|\lambda|$ is replaced by a small one. Thus the next lemma follows.

Lemma 2.10. There exists an $r_{0}>0$ such that for any $r \in\left(0, r_{0}\right)$, there exist constants $\rho, \lambda^{\prime}>0$ which satisfy

$$
I_{\lambda}(u) \geq \rho \quad \text { when }\|\nabla u\|_{2}=r,|\lambda|<\lambda^{\prime} .
$$

The lemma above will be used to find a small positive solution of (1.1), (1.2). We are now in a position to prove Theorem 1.1

Proof of Theorem 1.1. Choose $\lambda_{0}>0$ which satisfies Lemmas 2.7 and 2.9. Let $u_{\lambda}$ be a mountain pass solution of $I_{\lambda}$ with $|\lambda| \leq \lambda_{0}$. Then $0<u_{\lambda}(x) \leq 2 M$ by Lemma 2.9. Thus $h^{\prime}\left(u_{\lambda}\right)=0, h\left(u_{\lambda}\right)=1, \widetilde{g}\left(x, u_{\lambda}\right)=g\left(x, u_{\lambda}\right)$ and therefore $u_{\lambda}$ becomes a solution of (1.1), (1.2). Let $\lambda_{j}$ be any sequence converging to zero. By Lemma 2.8, a subsequence $u_{\lambda_{j}^{\prime}}$ converges to a mountain pass solution $u_{0}$ of $I_{0}$ in $W^{2, q}(\Omega)$ for all $q \in[1, \infty)$.

We now suppose that $g(x, 0) \geq 0, g(x, 0) \not \equiv 0$ in $\Omega$. By (2.6), we have

$$
\inf _{\|\nabla u\|_{2}=r} I_{\lambda}(u) \geq \rho / 2>0=I_{\lambda}(0) .
$$

Let $B$ be the set of $u \in H_{0}^{1}(\Omega)$ such that $\|\nabla u\|_{2} \leq r$. Then the minimum of $I_{\lambda}$ in $B$ is achieved at an interior point $v_{\lambda}$. Indeed, choose a sequence $u_{n}$ in $B$ such that $I_{\lambda}\left(u_{n}\right)$ converges to the infimum of $I_{\lambda}$ in $B$. A subsequence of $u_{n}$ weakly converges in $H_{0}^{1}(\Omega)$ to a point $v_{\lambda}$ in $B$. By the weakly lower semicontinuity of $I_{\lambda}$, we have

$$
I_{\lambda}\left(v_{\lambda}\right) \leq \liminf _{n \rightarrow \infty} I_{\lambda}\left(u_{n}\right)
$$

which means that $v_{\lambda}$ is a minimum point of $I_{\lambda}$ in $B$. Since $I_{\lambda}(0)=0$, we have $I_{\lambda}\left(v_{\lambda}\right) \leq 0<I_{\lambda}\left(u_{\lambda}\right)$, where $u_{\lambda}$ is a mountain pass solution of $I_{\lambda}$. Therefore $v_{\lambda} \neq u_{\lambda}$. In the same way as in Lemma 2.5 with $|\lambda|$ and $r>0$ small enough, we can prove that $\left\|v_{\lambda}\right\|_{\infty} \leq M$. Hence $\widetilde{g}\left(x, v_{\lambda}\right)=g\left(x, v_{\lambda}\right)$ and $v_{\lambda}$ is a solution of (1.1). Moreover, $v_{\lambda} \not \equiv 0$ because $g(x, 0) \not \equiv 0$. Thus $v_{\lambda}$ is a nontrivial solution. We shall show that $v_{\lambda}(x) \geq 0$ for $\lambda>0$. Let $D$ be the set of $x \in \Omega$ such that $v_{\lambda}(x)<0$. Since $f(x, s)=f(x, 0)=0$ and $g(x, s)=g(x, 0) \geq 0$ for $s<0$, we see that for $\lambda>0$,

$$
-\Delta v_{\lambda}=f\left(x, v_{\lambda}\right)+\lambda g\left(x, v_{\lambda}\right) \geq 0 \quad \text { in } D, \quad v_{\lambda}=0 \text { on } \partial D
$$

which shows that $v_{\lambda} \geq 0$ in $D$, a contradiction to the definition of $D$. Thus $D$ must be empty, and $v_{\lambda}(x) \geq 0$ in $\Omega$. By Lemma 2.10 $\left\|\nabla v_{\lambda}\right\|_{2} \rightarrow 0$ as $\lambda \rightarrow 0$. By the 
bootstrap argument, the $W^{2, q}(\Omega)$ norm of $v_{\lambda}$ converges to zero for all $q \in[1, \infty)$, and hence $v_{\lambda} \rightarrow 0$ in $C^{1}(\bar{\Omega})$. Then Lemma 2.9 (ii) shows that $v_{\lambda}(x)<u_{\lambda}(x)$ in $\Omega$ for $\lambda>0$ small enough.

We suppose that (1.3) holds. Put $A:=\left\|v_{\lambda}\right\|_{\infty}$. By (1.3), there is a $C>0$ such that

$$
g(x, s)-g(x, 0) \geq-C s \text { for } 0 \leq s \leq A, x \in \Omega .
$$

Moreover, $f(x, s) \geq-C s$ for $0 \leq s \leq A$ in the proof of Lemma 2.1. Then we have

$$
\begin{aligned}
((1+\lambda) C-\Delta) v_{\lambda}= & f\left(x, v_{\lambda}\right)+C v_{\lambda} \\
& +\lambda\left(g\left(x, v_{\lambda}\right)-g(x, 0)+C v_{\lambda}\right)+\lambda g(x, 0) \\
\geq & 0 .
\end{aligned}
$$

By the strong maximum principle, $v_{\lambda}$ is strictly positive. The proof is complete.

\section{REFERENCES}

[1] G. A. Afrouzi and K.J. Brown, Positive mountain pass solutions for a semilinear elliptic equation with a sign-changing weight function, Nonlinear Anal. 64 (2006), 409-416. MR2191987

[2] S. Alama and G. Tarantello, On semilinear elliptic equations with indefinite nonlinearities, Calc. Var. Partial Differential Equations 1 (1993), 439-475. MR1383913 (97a:35057)

[3] A. Ambrosetti and P. H. Rabinowitz, Dual variational methods in critical point theory and applications. J. Funct. Anal. 14 (1973), 349-381. MR0370183 (51:6412)

[4] K. J. Brown and Y. Zhang, The Nehari manifold for a semilinear elliptic equation with a sign-changing weight function, J. Differential Equations 193 (2003), 481-499. MR.1998965 (2004i:35069)

[5] R. Kajikiya, Mountain pass theorem in ordered Banach spaces and its applications to semilinear elliptic equations. Nonlinear Differential Equations and Applications 19 (2012), 159-175. MR.2902185

[6] S. Li and Z.-Q. Wang, Mountain pass theorem in order intervals and multiple solutions for semilinear elliptic Dirichlet problems, J. Anal. Math. 81 (2000), 373-396. MR.1785289 (2001h:35063)

[7] P. H. Rabinowitz, "Minimax methods in critical point theory with applications to differential equations," CBMS Regional Conf. Ser. in Math. 65, Amer. Math. Soc., Providence, 1986. $\operatorname{MR} 845785(87 \mathrm{j}: 58024)$

[8] M. Struwe, Variational Methods, second edition, Springer, Berlin, 1996. MR.1411681 (98f:49002)

[9] M. Willem, Minimax Theorems, Birkhäuser, Boston, 1996. MR.1400007 (97h:58037)

Department of Mathematics, Faculty of Science and Engineering, Saga University, SAGA, 840-8502, JAPAN

E-mail address: kajikiya@ms.saga-u.ac.jp 